\title{
Intervenções de enfermagem ao idoso hospitalizado com risco de queda
}

RESUMO | Objetivo: comparar as intervenções de enfermagem referentes ao risco de queda no idoso hospitalizado registradas nos prontuários, com as intervenções e atividades propostas pela Nursing Intervention Classification. Método: estudo transversal, descritivo, com utilização da ferramenta mapeamento cruzado, realizado em um hospital universitário. Coleta de dados realizada em formulário próprio e preenchido a partir das evoluções registradas pelos enfermeiros em 67 prontuários de idosos classificados com risco de queda. As frasestermo foram escritos manualmente no formulário e depois sumarizados em planilha de Excel 2011. Aplicou-se estatística descritiva simples com o auxílio do programa Statistical Package for Social Sciences- SPSS 22.0 para retratar e analisar os dados. Resultados: ao se comparar as intervenções registradas pelos enfermeiros com as Intervenções e Atividades de Enfermagem da Nursing Intervention Classification, evidenciaram-se 10 intervenções. Conclusão: $O$ estudo possibilitou a qualificação e otimização do cuidado em relação à prevenção de quedas, norteando e aperfeiçoando os planos terapêuticos de enfermagem. Através da articulação dos dados e conhecimentos gerados com o executar do estudo, pode-se conhecer e aprender acerca da diversidade de atividades sugeridas pelas intervenções/atividades NIC, as quais podem incrementar o registro de enfermeiro em relação às ações preventivas no evento de quedas.

Palavras-chaves: Idoso; Acidentes por Quedas; Cuidados de Enfermagem.

\begin{abstract}
Objective: to compare the nursing interventions related to the risk of falling in the hospitalized elderly recorded in the medical records, with the interventions and activities proposed by the Nursing Intervention Classification. Method: cross-sectional, descriptive study using the cross-mapping tool, carried out in a university hospital. Data collection was performed in its own form and filled out from the evolutions recorded by nurses in 67 medical records of elderly classified as at risk of falling. The term phrases were written manually on the form and then summarized in Excel 2011 spreadsheet. Simple descriptive statistics were applied with the aid of the Statistical Package for Social Sciences- SPSS 22.0 program to portray and analyze the data. Results: when comparing the interventions recorded by nurses with nursing interventions and activities of the Nursing Intervention Classification, 10 interventions were evidenced. Conclusion: The study enabled the qualification and optimization of care in relation to the prevention of falls, guiding and improving the therapeutic plans of nursing. Through the articulation of the data and knowledge generated with the execution of the study, one can know and learn about the diversity of activities suggested by NIC interventions/ activities, which can increase the nurse's record in relation to preventive actions in the event of falls.
\end{abstract}

Keywords: Elderly; Accidents Due to Falls; Nursing Care.

RESUMEN I Objetivo: comparar las intervenciones de enfermería relacionadas con el riesgo de caer en los ancianos hospitalizados registrados en los registros médicos, con las intervenciones y actividades propuestas por la Clasificación de Intervención de Enfermería. Método: estudio transversal y descriptivo utilizando la herramienta de mapeo cruzado, realizado en un hospital universitario. La recopilación de datos se realizó en su propia forma y se rellenó de las evoluciones registradas por las enfermeras en 67 registros médicos de ancianos clasificados como en riesgo de caída. Las frases del término se escribieron manualmente en el formulario y luego se resumieron en la hoja de cálculo de Excel 2011. Se aplicaron estadísticas descriptivas simples con la ayuda del programa paquete estadístico para ciencias sociales- SPSS 22.0 para representar y analizar los datos. Resultados: al comparar las intervenciones registradas por las enfermeras con las intervenciones de enfermería y las actividades de la Clasificación de Intervención de Enfermería, se evidenciaron 10 intervenciones. Conclusión: El estudio permitió la calificación y optimización de la atención en relación con la prevención de caídas, orientando y mejorando los planes terapéuticos de la enfermería. A través de la articulación de los datos y conocimientos generados con la ejecución del estudio, se puede conocer y conocer la diversidad de actividades sugeridas por las intervenciones/actividades NIC, que pueden aumentar el historial del enfermero en relación con las acciones preventivas en caso de caídas.

Palavras claves: Ancianos; Accidentes Debidos a Caídas; Cuidados de Enfermería.

\section{Eliane da Silva Pereira}

Mestre em Ciências do Cuidado e da Saúde. Universidade Federal Fluminense. RJ/Brasil.

\section{Selma Petra Chaves Sá}

Pós-Doutora em Enfermagem. Universidade Federal Fluminense; Departamento de Fundamentos de Enfermagem e Administração da Escola de Enfermagem Aurora de Afonso Costa. RJ/Brasil.

Recebido em: 01/06/2020

Aprovado em: 03/06/2020

\section{Anamaria Alves Napoleão}

Doutora em Enfermagem. Universidade Federal de São Carlos, Departamento de Enfermagem. SP/Brasil.

\section{Ana Carla Dantas Cavalcanti}

Pós-Doutora em Enfermagem. Universidade Federal Fluminense; Departamento de Fundamentos de Enfermagem e Administração da Escola de Enfermagem Aurora de Afonso Costa. RJ/Brasil.

\section{INTRODUÇÃO}

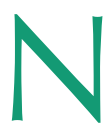

o Brasil, a população idosa é o segmento populacional que mais cresce nos últimos tem$\operatorname{pos}^{(1)}$. Tal crescimento pode ser justificado pelo controle da natalidade e aumento da expectativa de vida, resultado de maior oferta da qualidade de vida. Há previsões de que no período de 1950 a 2025, o grupo de idosos no país deverá ter aumentado em quinze vezes, enquanto a população total em cinco. Dessa forma, a nação de- 
verá ocupar o sexto lugar quanto ao contingente de idosos, alcançando, em 2025, cerca de 32 milhões de pessoas com 60 anos ou mais de idade ${ }^{(2)}$.

Um aspecto que preocupa em relação ao acelerado processo de envelhecimento são as repercussões nos serviços de saúde, a internação hospitalar é um importante recurso na atenção aos idosos, fazendo parte da rede de atenção à saúde. As hospitalizações, principalmente se repetidas e prolongadas, podem produzir consequências negativas à saúde dos idosos, como a diminuição da capacidade funcional, da qualidade de vida e o aumento da fragilidade ${ }^{(3)}$.

A hospitalização é compreendida pela sociedade como um período importante para a recuperação do paciente, entretanto, nem por isso é bem vinda por estar vinculada ao adoecimento e pode, consequentemente, potencializar o agravamento de certas condições físicas, em especial na população idosa, e predispor a ocorrência de eventos adversos, como as quedas.

As quedas são definidas, comumente, como "vir a inadvertidamente ficar no solo ou em outro nível inferior, excluindo mudanças de posição intencionais para se apoiar em móveis, paredes ou outros

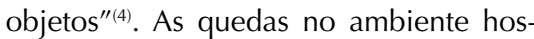
pitalar apresentam-se como o terceiro evento adverso mais notificado pelo Sistema Notivisa da Agência Nacional de Vigilância Sanitária (ANVISA). Os dados oriundos desse sistema apontam que de março de 2014 a março de 2017, mais de 12 mil quedas foram notificadas e, na sua maioria, por falta de equilíbrio ${ }^{(5)}$.

As quedas sofridas por pacientes durante o período de internação são intercorrências relevantes que demonstram a deficiência da segurança no cuidado, além de se constituírem nos serviços de saúde uma das preocupações prioritárias ao se discutir sistemas de controle de qualidade assistencial ${ }^{(6)}$.

O diagnóstico de enfermagem (DE) Risco de Quedas, proposto pela NANDA$-I$, atende em grande parte à necessidade de identificar o idoso de risco para propor e adicionar medidas que garantam sua integridade. A taxonomia da NANDA-I descreve pela primeira vez o DE Risco de Quedas no ano de 2000, inserido no domínio Segurança/Proteção, na classe Lesão Física. É definido como a suscetibilidade aumentada para quedas que podem causar danos físicos, os seus fatores de risco são diversos ${ }^{(7)}$.

Conforme a Nursing Interventions Classification (NIC), na intervenção Prevenção de Quedas, estão incluídas dez atividades relacionadas à orientação e ensino do paciente/família. Nas prescrições de enfermagem para os pacientes com DE Risco de Quedas, geralmente identifica-se a orientação do paciente/família quanto aos riscos e prevenção de quedas. São frequentes os cuidados prescritos pelos enfermeiros ${ }^{(8)}$.

Atrelado a esta questão do envelhecimento, o processo de hospitalização do idoso e os eventos adversos que podem acontecer nesse meio, em especial as quedas, há um despertar para a questão da segurança do paciente. O Brasil é um dos países que compõem a Aliança Mundial para a Segurança do Paciente e, em 2013, o Ministério da Saúde (MS) elaborou e divulgou a Portaria n. ${ }^{\circ}$ 529, que instituiu o Programa Nacional de Segurança do Paciente (PNSP), a qual define os conceitos relevantes na área e as principais estratégias para a implementação do programa. Dentre as estratégias para redução dos incidentes de segurança estabelecidas pelo PNSP, está o protocolo de prevenção de quedas voltado para o ambiente hospitalar ${ }^{(9)}$.

Com enfoque na Meta 6 da PNSP, que visa a prevenção de quedas e úlceras por pressão, ressalta-se que a hospitalização é apontada como um dos fatores que aumentam o risco de queda ${ }^{(10)}$. Este risco é mais acentuado em idosos, em razão do ambiente desconhecido, presença de várias doenças sistêmicas, submissão a procedimentos terapêuticos, uso de diversos medicamentos, além da fragilidade decorrente da morbidade que desencadeou a internação ${ }^{(11)}$.

O enfermeiro, sendo conhecedor das modificações que o processo de enveIhecimento acarreta, precisa estar atento para identificá-las, assim como perceber as necessidades expressas ou não, e determinar as ações para uma melhor qualidade de vida aos idosos, realizando cuidado individualizado, tentando manter a independência e autonomia ${ }^{(12)}$.

Sendo assim, a prevenção das quedas encontra-se articulada com a questão da implementação e da aplicação de intervenções eficazes por parte do enfermeiro e sua equipe, mantendo uma assistência segura e de qualidade.

Neste contexto, o estudo teve como objetivo comparar as intervenções de enfermagem referentes ao risco de queda no idoso hospitalizado registradas nos prontuários, com as intervenções e atividades propostas pela Nursing Intervention Classification (NIC).

\section{METODOLOGIA}

Trata-se de estudo transversal, documental e adotou a utilização da ferramenta mapeamento cruzado, realizado em um hospital público universitário em Niterói/ RJ. Optou-se em realizar a coleta no ambiente de Clínica Médica e Cirúrgica, feminina e masculina, a coleta de dados teve início no mês de maio de 2017 e encerrou no final do mês de julho de 2017. TrabaIhou-se com uma amostra de conveniência, que se constituiu de 67 prontuários de pessoas a partir dos 60 anos.

Como critérios de inclusão foram considerados os prontuários de: idosos que apresentassem período de internação superior a 48 horas; que o leito estivesse com a identificação visual para Risco de Queda; que no plano terapêutico, referente ao dia da internação, estivesse pontuado o Risco de Queda; e que, no mínimo, existissem duas evoluções redigidas pelo enfermeiro.

Já os critérios de exclusão considerados foram: os prontuários não localizados no local de guarda; e aqueles que, mesmo apresentando identificação visual no leito e identificados no plano terapêutico para risco de queda, não estavam redigidas nas evoluções as intervenções de enfermagem ligadas ao risco de queda.

As variáveis independentes sociodemo- 
Pereira, E.S.; Sá, S.P.C.; Napoleão, A.A · Cavalcanti, A.C.D · Intervenções de enfermagem ao idoso hospitalizado com risco de queda

gráficas identificadas foram: idade, sexo, renda, nível de escolaridade e estado civil.

Realizou-se o levantamento dos prontuários referentes aos idosos internados nas clínicas eleitas que atendiam aos critérios do estudo. A partir dos prontuários separados, identificaram-se nas evoluções as intervenções de enfermagem ligadas ao risco de queda descritas pelos enfermeiros para os idosos classificados com o tal risco, essas intervenções foram transcritas manualmente para um instrumento criado.

A partir do registro manual realizado no instrumento de coleta, elaborou-se um banco de dados com auxílio da planilha do Office Excel for Windows ${ }^{\circledR} 2011$, para efetuar o mapeamento das ações intervencionistas registradas. Devido às intervenções similares registradas na primeira e segunda evoluções e a aproximação verbal, considerou-se pertinente fazer um agrupamento por similaridade, demonstrado na Tabela 3 das ações intervencionistas mapeadas.

O mapeamento cruzado foi realizado por meio de comparação entre as ações redigidas nos prontuários, e as intervenções/atividades propostas pela NIC. Para realizar esse procedimento de mapeamento, fez-se necessário considerar as seguintes regras ${ }^{(13)}$ : mapear usando o contexto do diagnóstico de enfermagem; manter a consistência entre a intervenção sendo mapeada e a definição da intervenção NIC; mapear a intervenção partindo do rótulo da intervenção NIC para a atividade; usar a "palavra-chave" na intervenção, para mapear para a intervenção NIC; e usar os verbos como as "palavras-chave" na intervenção.

A partir dos dados coletados, foi construído um banco de dados em planilha do Excel Microsoft@ 2011. As análises estatísticas foram processadas por meio do programa IBM SPSS (Statistical Packagefor the Social Science), versão 22.0. Para caracterização da amostra e análise descritiva das variáveis, os dados sociodemográficos foram sintetizados em distribuições de frequências absolutas (n) e relativas (\%). Ainda na análise descritiva, realizou-se uma comparação percentual (\%) do total de atividades NIC relacionadas à queda identificadas nas intervenções mapeadas com as intervenções que foram encontradas nos registros pesquisados.

Em consonância com a Resolução n. ${ }^{\circ}$ 466/12/2012, do Conselho Nacional de Saúde (CNS), esta pesquisa foi encaminhada e aprovada pelo Comitê de Ética em Pesquisa (CEP) do Hospital Universitário Antônio Pedro (HUAP), sob o número de Protocolo CAAE 64103616.1.0000.5243 e
Parecer n. ${ }^{\circ}$ 2.004.425.

\section{RESULTADOS}

Com relação os dados sociodemográficos sintetizados, a partir das informações obtidas dos 67 prontuários inclusos no estudo, observa-se a frequência expressa na Tabela 1, onde nota-se o predomínio de idosos do sexo masculino, casados, com uma faixa etária de 60 a 64 anos.

Tabela 1. Distribuição de frequências das variáveis do perfil sociodemográfico UFF/ EEAAC/MACCS. Niterói, RJ, Brasil, 2017.

\begin{tabular}{lc}
\hline \multicolumn{1}{c}{ Variável } & $\begin{array}{c}\text { Frequência } \\
(\%)\end{array}$ \\
\hline Sexo & $22(32,8 \%)$ \\
Feminino & $45(67,2 \%)$ \\
Masculino & \\
Estado Civil & $22(32,8 \%)$ \\
Solteiro & $31(46,3 \%)$ \\
Casado & $01(1,5 \%)$ \\
União Estável & $02(3,5 \%)$ \\
Div orciado & $11(6,4 \%)$ \\
Viúvo & \\
Escolarid ade & $23(34,3 \%)$ \\
Não Informada & $01(1,5 \%)$ \\
Analfabeto & $13(19,4 \%)$ \\
Ensino Fundamental Incompleto & $16(23,9 \%)$ \\
Ensino Fundamental Completo & $10(14,9 \%)$ \\
Ensino Médio Completo & $04(6,0 \%)$ \\
Ensino Superior & \\
Renda Men sal & $24(35,8 \%)$ \\
Não Informada & $34(50,7 \%)$ \\
1 a 3 salários mínimos & $09(13,4 \%)$ \\
3 a 6 salários mínimos & $16(24,6 \%)$ \\
Id ade & $14(21,5 \%)$ \\
60 a 64 anos & $07(10,8 \%)$ \\
65 a 68 anos & $11(16,9 \%)$ \\
69 a 72 anos & $06(9,2 \%)$ \\
73 a 76 anos & $05(7,7 \%)$ \\
77 a 80 anos & $06(9,2 \%)$ \\
81 a 84 anos & \\
85 a 89 anos & \\
\hline &
\end{tabular}

Fonte: Pereira ES, 2018 ${ }^{(14)}$ 
Identificou-se no primeiro registro 176 frases-termo que expressavam intervenções de enfermagem relacionadas à prevenção do risco de queda.
Já o segundo registro apresentou um total de 112 frases-termos. Foram analisadas duas evoluções consecutivas redigidas pelos enfermeiros pós-ad-

Tabela 2. Distribuição de frequências de frases mapeadas dos registros. UFF/EEAAC/ MACCS. Niterói, RJ, Brasil, 2017.

\begin{tabular}{|c|c|c|}
\hline Registros & $\mathrm{N}$ & $\begin{array}{c}\text { Frequência } \\
(\%)\end{array}$ \\
\hline \multicolumn{3}{|l|}{ Primeiro Registro } \\
\hline Leito mantido com grades elevadas & 45 & $67,2 \%$ \\
\hline Avaliado nivel de consciência & 44 & $65,7 \%$ \\
\hline Encaminhado ao banho de aspersão com auxílio & 23 & $34,3 \%$ \\
\hline Deambula com auxílio & 19 & $28,4 \%$ \\
\hline Auxílio de cadeira higiênica e de rodas & 14 & $20,9 \%$ \\
\hline Paciente restrito ao leito & 13 & $19,4 \%$ \\
\hline Orientado a família sobre auxiliar o paciente & 13 & $19,4 \%$ \\
\hline Movimentação em bloco e posicionamento no leito & 05 & $7,5 \%$ \\
\hline \multicolumn{3}{|l|}{ Segundo Registro } \\
\hline Avaliado nível de consciência & 35 & $52,2 \%$ \\
\hline Repouso no leito com grades elevadas & 27 & $40,3 \%$ \\
\hline Auxilio para deambular em pequenas distâncias & 10 & $14,9 \%$ \\
\hline Orientação do familiar para mobilização no leito & 09 & $13,4 \%$ \\
\hline Encaminhado ao banho de cadeira higiênica & 07 & $10,4 \%$ \\
\hline Identificação no leito de risco de queda & 07 & $10,4 \%$ \\
\hline Paciente restrito ao leito & 06 & $9,0 \%$ \\
\hline Contido no leito & 05 & $7,5 \%$ \\
\hline Banho no leito & 03 & $4,5 \%$ \\
\hline $\begin{array}{l}\text { Orientado o paciente como movimentar-se com } \\
\text { auxílio do andador/muleta }\end{array}$ & 02 & $3,0 \%$ \\
\hline Avaliar equilíbrio & 01 & $1,5 \%$ \\
\hline
\end{tabular}

Fonte: Pereira ES, 2018 ${ }^{(14)}$

Tabela 3. Distribuição da frequência dos Grupos Similares. UFF/EEAAC/MACCS. Niterói, RJ, Brasil, 2017.

\begin{tabular}{lcc}
\hline \multicolumn{1}{c}{ G rupos de Similares } & N & $\begin{array}{c}\text { F requência } \\
\mathbf{( \% )}\end{array}$ \\
\hline Nivel de Con sciência & 80 & $59,7 \%$ \\
Leito G radeado & 72 & $53,7 \%$ \\
Auxílio na lo comoção e/ou transferên cia & 55 & $41,0 \%$ \\
Orien tação ao familiar e/ou paciente & 24 & $17,9 \%$ \\
Condiça do paciente & 24 & $17,9 \%$ \\
Risco de Queda informado & 07 & $5,2 \%$ \\
\hline
\end{tabular}

Fonte: Pereira ES, 2018

missão dos pacientes, de 67 prontuários lidos, totalizaram-se 134 evoluções lidas, culminando assim em 288 frases-termos mapeados.

No primeiro registro, as maiores frequências dos termos identificados foram: leito mantido com grades elevadas (45 casos, 67,2\%) e avaliado nível de consciência (44 casos, 65,7\%). Já no segundo registro, as maiores frequências de termos mapeados foram: nível de consciência (35 casos, 52,2\%) e repouso no leito com grades elevadas (27 registros, 40,3\%). Outros termos também foram mapeados, como se visualiza nos registros da Tabela 2 .

Com as intervenções mapeadas na $1^{\mathrm{a}}$ e $2^{\mathrm{a}}$ evoluções, procedeu-se a um agrupamento por similaridade das intervenções, demonstrado na Tabela 3, para que fosse feita a comparação do que se encontrou nos registros com o que a literatura, a NIC, apresenta.

Conforme os dados apresentados nas Tabelas 2 e 3, elencaram-se 10 Intervenções e 37 atividades mapeadas relacionadas à Classificação de Intervenção de Enfermagem (NIC), a seguir apresentadas: Controle do Ambiente; Assistência no Autocuidado: banho/higiene; Assistência no Autocuidado: transferência; Assistência no AUTOCUIDADO: uso de vaso sanitário; Banho; CONTENÇÃO Física; POSICIONAMENTO: cadeira de rodas; Prevenção contra QUEDAS; Cuidados com o REPOUSO no Leito e TRANSFERÊNCIA. Das intervenções NIC mapeadas, $40 \%$ apresenta-se ligada ao Domínio Fisiológico: Básico e o Nível: Facilitação do Autocuidado.

Quadro 1. Intervenções e Atividades de Enfermagem segundo a NIC, mapeadas nos registros interligados com os Grupos Similares formados. UFF/EEAAC/MACCS. Niterói, RJ, Brasil, 2017

\begin{tabular}{|l|l|l|}
\hline Intervenção NIC & \multicolumn{1}{|c|}{ Atividade NIC } & \multicolumn{1}{|c|}{ Intervenção Registrada } \\
\hline $\begin{array}{l}\text { Controle do AM- } \\
\text { BIENTE: segurança }\end{array}$ & $\begin{array}{l}\text { Identificar as necessidades de segurança do paciente, com base no nível de capacidade } \\
\text { fisíca e cognitiva e no histórico comportamental anterior. } \\
\text { Usar dispositivos protetores para limitar fisicamente, a mobilidade ou o acesso a situa- } \\
\text { ções prejudiciais. }\end{array}$ & $\begin{array}{l}\text { Avaliar nível de consciência. } \\
\text { Conter paciente. }\end{array}$ \\
\hline $\begin{array}{l}\text { Assistência no } \\
\text { AUTOCUIDADO: } \\
\text { banho/higiene }\end{array}$ & $\begin{array}{l}\text { Determinar quantidade e tipo de assistência necessária. } \\
\text { Facilitar que o paciente tome o banho sozinho, conforme apropriado. }\end{array}$ & $\begin{array}{l}\text { Encaminhar ao banho de } \\
\text { aspersão. } \\
\text { Realizar banho no leito }\end{array}$ \\
\hline
\end{tabular}


Pereira, E.S.; Sá, S.P.C.; Napoleăo, A.A.; Cavalcanti, A.C.D.; Intervençōes de enfermagem ao idoso hospitalizado com risco de queda

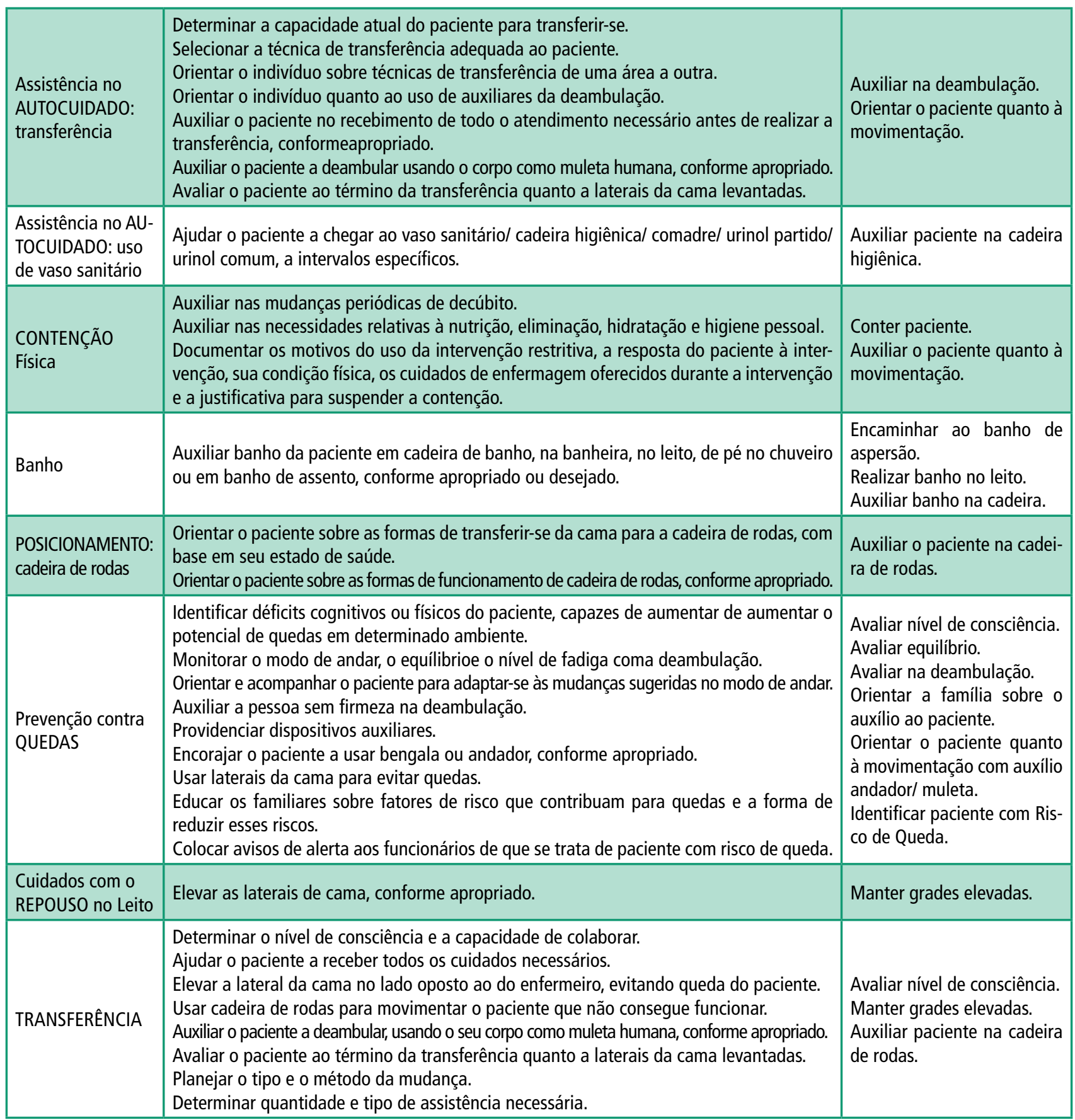

Fonte: Pereira ES, 2018

\section{DISCUSSÃO}

Primeiramente, é de extrema importância entender que é mediante o registro que se respalda tudo que foi realizado no processo assistencial pelo enfermeiro e sua equipe. Os debates em torno dos registros escritos pela enfer- magem apontam para sua apresentação como um dos principais instrumentos de comunicação para a troca de informações entre as equipes envolvidas no cuidado, cujo objetivo reside em apresentar de forma clara as necessidades do paciente, as condutas clínicas de cuidar que foram implementadas e a avaliação contínua do cuidado prestado. Nesse aspecto, registrar é uma responsabilidade ética e social ${ }^{(14)}$.

Há publicações que explicitam a importância e quais são as medidas preventivas redigidas em relação à queda 
no ambiente hospitalar, como as que foram apresentadas em um estudo referentes às medidas preventivas em relação à queda no idoso, inferem que em artigos publicados de 2002 a 2012: programas de prevenção de quedas; ferramentas de avaliação de risco; diretrizes; intervenções; presença de acompanhante/ voluntário; diminuição do trabalho enfermagem; escalas de avaliação de funcionalidade e motora; luz de chamada; cuidados de enfermagem; identificação de risco de queda; exercícios; restrição; auxílio à deambulação; presença de grades laterais do leito; alarme; cama baixa e calçados adequados, foram ações intervencionistas redigidas, a fim da manutenção da segurança do paciente em relação a queda ${ }^{(15)}$.

Na prática clínica da enfermagem é fundamental uma avaliação do risco de queda, assente na natureza multifatorial deste evento. Sendo desejável a utilização das classificações e linguagens padronizadas, que representam o conhecimento atual. O enfermeiro com informação relevante para um diagnóstico acurado pode intervir, redigir cuidados necessários de forma adequada, visando resultados positivos na saúde dos idosos ${ }^{(16)}$.

Neste presente estudo, executou-se um agrupamento representado na Tabela 3, apresentado nos resultados, dos Grupos Similares, das ações descritas pelos enfermeiros e mapeadas, promovendo uma facilitação no processo de comparação entre o que evidenciou-se no registro e o que a NIC apresenta, possibilitando ainda a detecção das intervenções mais destacadas numa avaliação geral dos registros.

Em relação ao mapeamento da questão de avaliação do nível de consciência, observou-se que tal termo foi descrito claramente em 59,7\% dos prontuários analisados para o estudo. Ao interligar/comparar a questão de avaliação do nível de consciência com as intervenções propostas na NIC, em consonância com as atividades, as intervenções identificadas foram: Contro- le do AMBIENTE: segurança, Prevenção contra QUEDAS e TRANSFERÊNCIA.

Em um estudo, sobre análise do diagnóstico risco de queda em paciente adulto hospitalizado, destacou-se a condição cognitiva do paciente como importante fator a ser considerado para a questão do risco de queda apresentando a relevância de avaliar e vigiar o sensório, o cognitivo, e também a avaliação e comunicação para toda a equipe de qualquer alteração nesses níveis ${ }^{(17)}$.

O maior número de quedas entre idosos com comprometimento cognitivo pode ser explicado pelo declínio em domínios cognitivos específicos, como função executiva, atenção e memória ${ }^{(18)}$.

O registro da manutenção do leito gradeado foi prescrito em 53,7\% nos prontuários estudados. Ao cruzar essa intervenção prescrita pelos enfermeiros com as traçadas pela NIC, obteve-se a ligação com as intervenções: controle do AMBIENTE: segurança, cuidados com o REPOUSO no leito e a TRANSFERÊNCIA. Essa ligação foi estabelecida de acordo com a própria essência da intervenção e as atividades apresentadas por elas.

Pesquisa refere que todos os leitos do ambiente, no qual o estudo foi realizado, possuem grades que abrem somente pelo lado de fora, induzindo o paciente a solicitar auxílio da equipe de enfermagem ou do acompanhante para abrir as grades ao sair do leito e enfatiza que a manutenção das grades elevadas é um fator de extrema importância para a proteção dos idosos no ambiente hospitalar ${ }^{(19)}$.

Sobre as intervenções registradas vinculadas à questão de auxílio na locomoção e/ou transferência, as intervenções semelhantes com a NIC identificadas foram: Assistência no AUTOCUIDADO: banho/higiene; Assistência no AUTOCUIDADO: transferência; Assistência no AUTOCUIDADO: uso de vaso sanitário, banho; POSICIONAMENTO: cadeira de rodas e TRANSFERÊNCIA.

São de suma importância a identificação e o registro da necessidade de auxílio na deambulação/transferência ao idoso hospitalizado, tendo em vista a redução da possibilidade da ocorrência das quedas, já que este idoso além de apresentar uma alteração no nível geral de sua saúde, também encontra-se em um novo espaço físico que, isoladamente, já se torna um ambiente de risco para executar sozinho certas atividades.

Destaca-se que ações como, "Auxiliar a pessoa sem firmeza na deambulação" e "Providenciar dispositivos auxiliares (p. ex., bengala e andador) para deixar o andar mais firme" são intervenções que mostram uma concordância de $100 \%$ entre as ações traçadas para a prevenção de quedas na aplicabilidade de intervenção de enfermagem da NIC em adultos e idosos hospitalizados ${ }^{(20)}$.

Sobre a orientação ao familiar e/ou paciente, identificou-se a descrição de tal ação em 17,9\% dos registros estudados, e as intervenções NIC associadas foram: Assistência no AUTOCUIDADO: transferência e Prevenção contra QUEDAS. Estas apresentavam atividades vinculadas à questão de orientação ao familiar e do paciente.

Ademais, é essencial que os profissionais de saúde se envolvam e redijam ações de educação em saúde, juntamente com os familiares dos idosos com potencial risco de queda, assim, favorecendo uma troca de informações no sentido de facilitar a identificação dos fatores de risco e a seleção de estratégias para a redução das quedas ${ }^{(4)}$.

O grupo similar, identificado como condição do paciente, foi composto pelas informações encontradas de como os pacientes apresentavam-se, por exemplo, "paciente contido" e "paciente restrito ao leito". Essas condições foram encontradas e descritas em $17,9 \%$ dos prontuários inclusos no estudo e, ao articular com as intervenções NIC, mapearam-se: Controle do AMBIENTE: segurança e CONTENÇÃO Física.

Ao analisar a questão da contenção física, a fim de prevenir a ocorrência de quedas em idosos hospitalizados, observa-se que a prevalência do registro de 
tal conduta não é alta. Dados similares foram encontrados em outro estudo(17), no qual entende-se que a implementação deste tipo de cuidado é muito utilizada em pacientes idosos semiconscientes, inconscientes e demenciados agitados, devendo sempre preservar a questão humanitária do paciente, seguindo os protocolos institucionais e parâmetros legais.

O uso da contenção física, na maioria dos casos, é implementado por razões de segurança, sendo a razão maior a prevenção de quedas. Ratificando ainda a necessidade da descrição clara da execução desta medida de segurança ${ }^{(21)}$.

Em relação à descrição sobre a identificação do risco de queda, ou seja, o risco de queda informado como foi nomeado no Grupo Similar, observou-se no estudo que, mesmo o enfermeiro e sua equipe detectando esse risco nos leitos, após avaliação prévia do paciente, essa intervenção foi pouco descrita nos prontuários, apenas 5,2\% apresentaram tal ação redigida.

Ao comparar o grupo similar risco de queda informado com a intervenção NIC e as atividades apresentadas, pode-se evidenciar que tal ação encontra-se diretamente relacionada à intervenção Prevenção contra QUEDAS, que destaca a importância da colocação de avisos de alerta aos funcionários de que se trata de um paciente com risco de queda e também o registro efetivo no prontuário dessa identificação.

Entende-se que a prevenção das quedas e a correta identificação dos pacientes que apresentam suscetibilidade para tal ocorrência são elementos que alicerçam a promoção da segurança do paciente; assim, o conhecimento do risco à queda pode significar um indicador para o monitoramento da segurança do paciente nesse aspecto ${ }^{(22)}$.

É pertinente elucidar que, em relação ao domínio NIC prevalecente no estudo em relação às intervenções NIC mapeadas, destaca-se: o Domínio Fisiológico: Básico e o Nível: Facilitação do Autocuidado. Essa informação apresenta uma in-

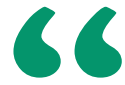

Entende-se que a prevenção das quedas e a correta identificação dos pacientes que apresentam suscetibilidade para tal ocorrência são elementos que alicerçam a promoção da segurança do paciente; assim, o conhecimento do risco à queda pode significar um indicador para o monitoramento da segurança do paciente nesse aspecto ferência, no que se visualiza no decorrer do estudo, que, apesar da prevenção de quedas ser uma questão que apresenta cuidados vinculados a outros domínios e níveis como Segurança: controle de risco e Fisiológico Básico: controle de imobilidade, os quais também afloraram no estudo, no entanto, a questão da facilitação, do auxílio e da ajuda no desempenhar das atividades do idoso hospitalizado são mais emergentes ao se pesquisar as ações efetuadas e registradas, tendo em vista a prevenção do evento queda hospitalar.

No desenvolver deste estudo documental e de mapeamento enfocando nas ações intervencionistas de prevenção de quedas, surgiram algumas limitações, dentre elas a dificuldade de coleta de dados, utilizando como fonte o prontuário do paciente e evolução do enfermeiro. Acredita-se que o presente estudo possa servir de base para o incremento de novas ações preventivas referentes ao evento de queda no plano terapêutico de enfermagem. Almeja-se também que novos estudos com a utilização do mapeamento cruzado sejam desenvolvidos, possibilitando ampliação no registro padronizado.

\section{CONCLUSÃO}

Este estudo mapeou as intervenções de enfermagem registradas pelos enfermeiros para os idosos com risco de queda registradas nos prontuários, com as intervenções/atividades propostas pela Classificação das Intervenções de Enfermagem (NIC), mediante a ferramenta metodológica do mapeamento cruzado e constatou que as intervenções semeIhantes à NIC apresentavam-se redigidas pelos enfermeiros.

Ao comparar as intervenções de enfermagem registradas nos prontuários com as propostas pela NIC, reconheceu-se a complexidade do cuidado com os idosos hospitalizados, e inter-relacionado com a prevenção das quedas.

De todas as 288 frases intervencionistas mapeadas, ligadas à prevenção de 
quedas, nos 67 prontuários analisados, pôde ser feito um agrupamento das intervenções semelhantes, o que culminou em 06 grupos identificados no decorrer do estudo como Grupos de Similares.

É de grande valia destacar que a intervenção Prevenção contra QUEDAS, que está diretamente ligada ao diagnóstico de Risco de Queda, foi a que obteve maior quantitativo de atividades mapeadas e comparadas que se encontrou no registro dos enfermeiros.

Com o realizar do estudo, houve a possibilidade de conhecer e aprender acerca da diversidade de atividades sugeridas pelas intervenções/atividades NIC, as quais podem incrementar o registro de enfermeiro em relação às ações preventivas no evento de quedas. É de suma importância desenvolver estudos que possam despertar a criação de instrumentos padronizados, protocolos, bundles, dentre outros, que disponibilizem maior quantitativo de intervenções pertinentes e eficazes na prevenção de quedas no ambiente hospitalar. Instrumentos esses que possam, além de agregar mais ações intervencionistas, contribuir para um processo de registro mais ágil e que contemplem os fatores importantes a serem destacados.

Assim, o estudo reafirma a necessidade da realização de pesquisas que sustentem a prática do cuidado associado à questão da manutenção da segurança do paciente, em especial à questão de ações preventivas para a fim de evitar a ocorrência de quedas na população idosa hospitalizada.

\section{Referências}

1. Silva Júnior FJG, et al. Risk of falls among hospitalized elderly: tool for patient safety. Rev Enferm UFPI. 2015 Oct-Dec;4(4):75-81.

2. Ministério do Planejamento, Orçamento e Gestão (BR); Instituto Brasileiro de Geografia e Estatística - IBGE. Sinopse do Censo Demográfico de 2010. Rio de Janeiro: IBGE; 2011. 265 p.

3. Dutra MM, Moriguchi EH, Lampert MA, Poli-de-Figueiredo CE. Validade preditiva de instrumento para identificação do idoso em risco de hospitalização. Rev saúde pública [Internet]. 2011;45(1):106-12. Doi: https://doi.org/10.1590/S0034-89102011000100012.

4. Organização Mundial de Saúde, Secretaria de Estado da Saúde(SP). Relatório global da OMS sobre prevenção de quedas na velhice.Campos LM, tradutor. [Internet], 2010 [cited 2014 set 10]. Available from: http:// bvsms.saude.gov.br/bvs/publicacoes/relatorio_prevencao_quedas_veIhice.pdf.

5. Proqualis (BR) [Internet]. Rio de Janeiro: Fiocruz; c2017-2018 [citado 2017 ago 05]. Breves I. Queda é um dos eventos adversos evitáveis mais notificados no país; [aprox. 2 telas]. Disponível em: https://proqualis. net/noticias/queda-\%C3\%A9-um-dos-eventos-adversos-evit $\% C 3 \% \mathrm{~A}$ 1 veis-mais-notificados-no-pa\%C3\%ADs.

6. Bausch AB, Waterkemper R, Linch GFC, Paz AA, Pelegrini AHW. Mortality due to falls from hospital beds: a retrospective study. Rev Baiana Enferm [Internet]. 2017 [citado 2018 mar 15];31(2):e17023. Disponível em: https://portalseer.ufba.br/index.php/enfermagem/article/downlo$\mathrm{ad} / 17023 / 14516$

7. NANDA Internacional. Diagnósticos de enfermagem da NANDA: definições e classificação 2012-2014. Garcez RM, tradutor. Porto Alegre: Artmed; 2013.

8. Bulechek GM, Butcher HK, Dochterman JM, Wagner CM. NIC Classificação das intervenções de enfermagem. 6. ed. Rio de Janeiro: Elsevier; 2016.

9. Brasil. Agência Nacional de Vigilância Sanitária (ANVISA) Ações de vigilância sanitária para segurança do paciente em serviços de saúde [Internet]. 2013 [cited 2016 Jul 16]. Available from: http://portal.anvisa. gov.br.

10. Ministério da Saúde (BR). Portaria n. ${ }^{\circ} 529$, de $1^{\circ}$ de abril de 2013. Institui o Programa Nacional de Segurança do Paciente (PNSP) [Internet]. Diário Oficial [da] União, Brasília, DF, 2 abr 2013 [cited 2016 dez 02]. Available from: http://bvsms.saude.gov.br/bvs/saudelegis/gm/2013/ prt0529_01_04_2013.html.

11. Vaccari $E$, Lenardt $M H$, Willig $M H$, Betiolli SE, Oliveira ES. Safety of the hospital environment in terms of preventing falls on the part of the elderly: a descriptive study. Online braz j nurs [Internet]. 2014;13 (3):271-81. Doi: https://doi.org/10.5935/1676-4285.20144753

12. Resende JO, Silva FMR, Assunção RS, Quadros KAN. Assistência do enfermeiro ao idoso na estratégia saúde da família. Rev enferm Cent-0este Min [Internet]. 2015;5(3):1831-43. Doi: http://dx.doi.org/10.19175/ recom.v5i3.880.

13. Lucena AF, Barros ALBL. Mapeamento cruzado: uma alternativa para a análise de dados em enfermagem. Acta paul enferm [Internet]. 2005;18(1):82-8. Doi: http://dx.doi.org/10.1590/5010321002005000100011.

14. Diniz SOS, Silva PS, Figueiredo NMA, Tonini T. Qualidade dos registros de enfermagem: reflexões analíticas em suas formas e conteúdos. Rev enferm UFPE on-line [Internet]. 2015;9(10):9616-23. Doi: 10.5205/reuol. 7944-69460-1-SM.0910201526,

15. Mello BLD. Quedas de pacientes em instituições hospitalares: uma revisão integrativa da literatura [Dissertação]. Ribeirão Preto (SP): Universidade de São Paulo (USP); 2013. [cited 2017 Mar 18]. 468 p. Available from: http://www.teses.usp.br/teses/disponiveis/22/22132/ tde-22012014-112444/pt-br.php.

16. Sousa LMM, Marques-Vieira CMA, Caldevilla MNGN, Henriques CMAD, Severino SSP, Caldeira SMA. Risco de quedas em idosos residentes na comunidade: revisão sistemática da literatura. Rev gaúcha enferm [Internet]. 2016 dez; 37(4):e55030. doi: http://dx.doi.org/10.1590/1983 1447.2016.04.55030.

17. Luzia MF, Almeida MA, Lucena AF. Mapeamento de cuidados de enfermagem para pacientes com risco de quedas na Nursing Interventions Classification. Rev esc enferm USP [Internet]. 2014;48(4):632-40. Doi: http://dx.doi.org/10.1590/S0080623420140000400009.

18. Cruz DT, Cruz FM, Ribeiro AL, Veiga CL, Leite ICG. Associação entre capacidade cognitiva e ocorrência de quedas em idosos. Cad saúde colet [Internet]. 2015; 23(4):386-93. Doi: http://dx.doi.org/10.1590/ 1414-462X201500040139.

19. Vaccari É. 0 evento queda em idosos hospitalizado [Dissertação] Curitiba (PR): Universidade Federal do Paraná; 2013. [cited 2017 Sep 03]. 137 p. Available from: http://acervodigital.ufpr.br/handle/1884/34833. 20. Oliveira JS. Risco de quedas: aplicabilidade de intervenções de Enfermagem da NIC em adultos e idosos hospitalizados [Tese]. Ribeirão Preto (SP): Universidade de São Paulo; 2013. 286 p. [cited 2019 Mar 31]. Available from: http://www.teses.usp.br/teses/disponiveis/22/22132/ tde-26092013-201408/pt-br.php.

21. Costa CPL. A enfermagem de reabilitação e os eventos adversos da restrição física da mobilidade [Dissertação]. Coimbra (PT): Escola Superior de Enfermagem de Coimbra; 2013. [cited 2019 Mar 31]. Available from: http://repositorio.esenfc.pt/?url=tc4FEopO.

22. Costa-Dias MJM, Ferreira PL. Fall risk assessment tools. Referência [Internet]. 2014; 4(2):153-61. Doi: http://dx.doi.org/10.12707/ RIII12145. 